\title{
Multi-fields model for predicting target-ligand interaction
}

\author{
Caihua Wang ${ }^{\mathrm{a}}$, Juan Liu ${ }^{\mathrm{a}, *}$, Fei Luo ${ }^{\mathrm{a}}$, Qian-Nan $\mathrm{Hu}^{\mathrm{b}, *}$ \\ ${ }^{a}$ School of Computer, Wuhan University, Wuhan 430072, PR China \\ ${ }^{b}$ Tianjin Institute of Industrial Biotechnology (TIB) of Chinese Academy of Sciences
}

(CAS), Tianjin 300308, PR China

\begin{abstract}
Predicting target-ligand interactions is a critical task of chemogenomics and plays a key role in virtual drug discovery. Moreover, it is important to take insights into the molecular recognition mechanisms between chemical substructures of ligands and binding sites of targets. In this work, we suppose the interaction between a ligand and a target is the result of the comprehensive effect of multiple fields between the ligand and the binding site of the target, and propose a multi-fields interaction model (MFIM) to predict the target-ligand interaction. The evaluation result on the same data set shows that MFIM outperforms other two representative methods. The derived fragment interaction network is robust to the parameter fluctuation and the connections in the network are sparse. Moreover, the edge weights of the network might reflect the fragment interaction intensity and most of the significant edges are chemical interpretable.
\end{abstract}

Keywords: Protein representation, Ligand representation, Field interaction, Target-ligand interaction, Target-ligand network analysis

\section{Introduction}

Target-ligand interactions play an initial role in cell signal conduction and predicting interactions between targets and ligands is the key task of chemogenomics. Through various high-throughput experimental projects for

${ }^{*}$ Corresponding author. Tel.\&Fax.: +86-027-68775711; Tel.\&Fax.:+86-022-24828786

Email address: liujuan@whu.edu.cn; hu_qn@tib.cas.cn (Qian-Nan Hu ) 
analyzing the genome, transcriptome and proteome, we are beginning to understand the genomic spaces populated by these protein classes. Meanwhile, the high-throughput screening of large-scale chemical compound libraries with various biological assays is enabling us to explore the chemical space $[1,2]$. However, our knowledge about the relationship between the chemical and genomic spaces is very limited [3, 4]. Since experimental ways to determine target-ligand interactions are costly and time-consuming, there is a strong incentive to develop computational methods capable of detecting potential target-ligand interactions efficiently.

Traditional computational methods could be roughly classified into two categories: ligand-based ones and target-based ones [3]. In ligand-based approaches, a ligand is compared to the ligands known interacting with a given target to make the prediction. Therefore, these approaches require enough number of ligands binding with the given target. Molecular docking is a typical target-based approach, however, it is time-consuming and is tricky to find a score function to select the best conformation [5].

Both of the above types of methods only consider information on one side of the interaction pair whereas ignoring important information on the other side. In recent years, researchers have begun to take the viewpoint of targetligand based chemogenomics approaches, taking information on both sides of the interaction pair into consideration. Bleakley and Yamanishi showed that the ensemble of ligand-based and target-based prediction models performed much better than only using single type of model [6]. Based on this fact, they proposed a bipartite local model (BLM). The BLM method has been further studied and improved by some researchers [7, 8, 9]. Leslie et al. first described the targets as full length sequences whose similarities were measured by the mismatch kernel $[10,11]$; and represented the ligands with PubChem fingerprints [12] whose similarities were measureed by the Tanimoto kernel. Then they constructed the pairwise support vector machine (pSVM) to predict the target-ligand interactions. Vert et al. followed Leslie's framework excepting that they adopted the local alignment kernel [13] to compare target similarity. Jacob et al. also followed Leslie's framework, however they described targets (proteins) as EC numbers, and applied a hierarchy kernel (tree-based kernel) to compare target similarity [14]. Van Laarhoven et al. represented targets as sequences and ligands with molecular graphs. They applied Smith-Waterman score [15] to measure the target sequence similarity, and used SIMCOMP [4] to measure the similarity score between moleculars. They also got the target-ligand interaction network profiles and adopted 
Gaussian kernel to measure the network profile similarity. After that, they combined the Smith-Waterman score and Gaussian kernel to get the final target kernel, and combined the SIMCOMP score and Gaussian kernel to get the final ligand kernel. And then, they used the kernel ridge regression (RLS) [7] to make a prediction. Yamanishi et al. developed a supervised learning algorithm to infer unknown drug-target interactions by integrating the chemical space and genomic space into a unified space ("pharmacological space"), in which the higher probabilities a target and ligand interact with each other, the closer they are [4]. Besides, many researchers employ data-driven approaches to predict target-ligand interaction, as availability of many genomic and chemogenomic data sources. Wang YC et al. proposed a kernel-based method to predict drug-protein interactions by integrating multiple types of data $[16,17,18]$. Zhao SW and Li S developed a computational framework, drugCIPHER, to infer drug-target interactions in a genome-wide scale [19].

Basic assumptions of most chemogenomic-based approach are twofold: compounds sharing some chemical similarity should also share targets and targets sharing similar ligands should share similar patterns/binding-sites [3]. Most of the previous work are based on the two assumptions $[6,7,8,9,10,13$, 14]. Although those methods usually performance well on the data set, we know little about the interaction mechanisms, which means the algorithms work in a black box. In this work, inspired by QSAR [20] which decomposes the interactions into different fields, we propose a new model called multifields interaction model (MFIM) to give insight into the molecular recognition mechanisms between chemical substructures of ligands and binding sites of targets. Our hypothesises are 1) there are multiple types of fields around a molecular fragment. The interaction of two molecular fragments is the result of the comprehensive effect of multiple fields; 2) the fields around a bindingsite/ligand originate in the superposition of the fields of their fragment. The target-ligand interaction is the result of the comprehensive effect of their fragment fields; 3 ) different types of fields are heterogeneous and heterogeneous fields can not interact with each other. For instance, the electrostatic field does not interact with hydrophobic field. Based on the three hypothesises, we build our algorithm (Figure 1). First, according to the target dictionary and the ligand dictionary, the binding sites of the targets and the ligands are encoded as binding site fragment vectors and ligand substructure vectors, forming the origin target and ligand spaces respectively. Then, we figure out the multiple fields intensities around the molecular fragments $(\boldsymbol{U}, \boldsymbol{V})$, 
and the two origin spaces are mapped into target and ligand field spaces respectively. The target fields would interact with the homogeneous ligand fields and a pairwise field space is generated. Finally, we construct a classifier to predict site-ligand interactions in the pairwise field space. Moreover, we integrate the multiple fields of molecular fragments and obtain a fragment interaction network, which give insight into the molecular recognition mechanisms between chemical substructures of ligands and binding sites of targets.

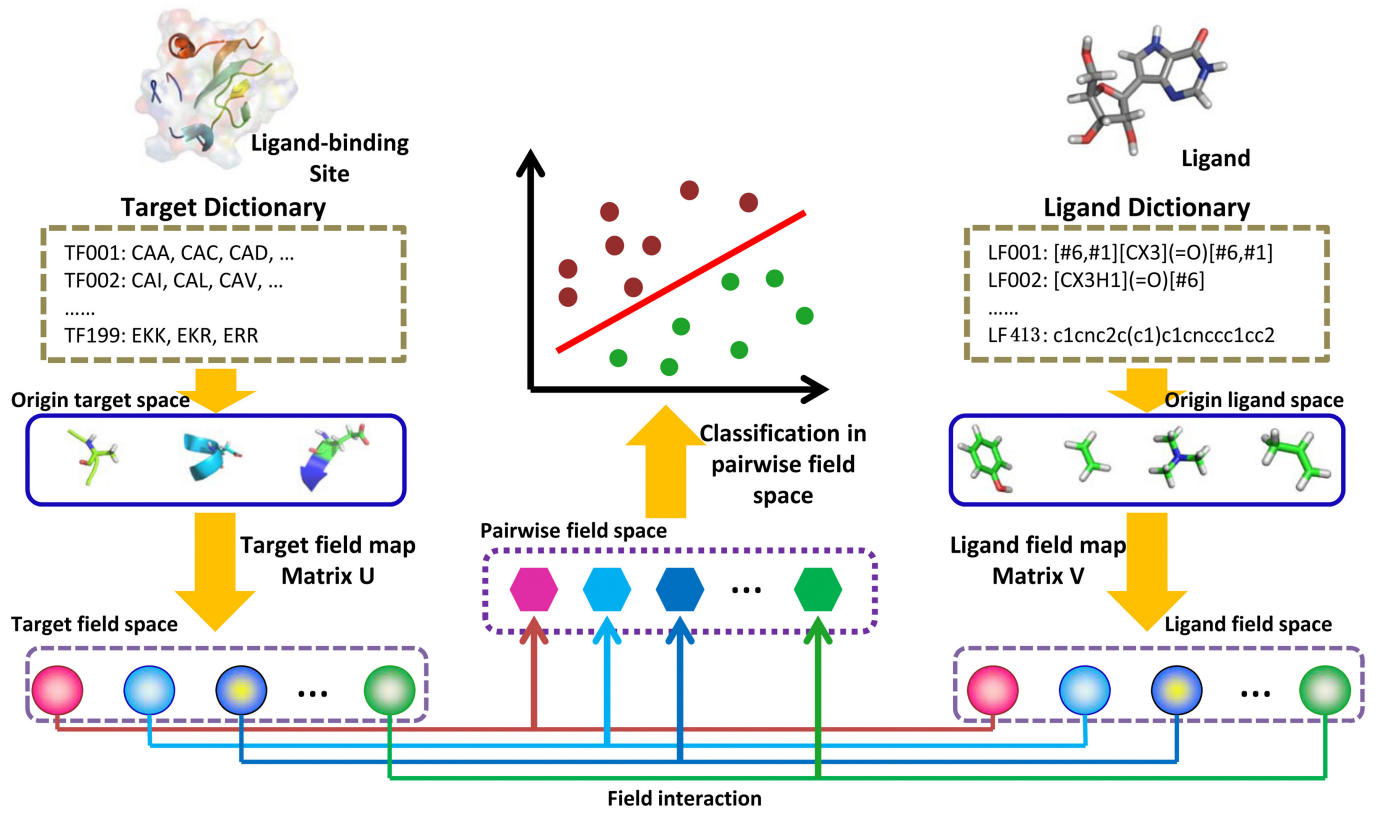

Figure 1: Multi-Field Interaction Model. First, based on the target dictionary and the ligand dictionary, the binding sites of the targets and the ligands are encoded as binding site fragment vectors and ligand substructure vectors, forming the target and ligand spaces respectively. Then, two spaces are mapped into target and ligand field spaces respectively. The target fields would interact with the homogeneous ligand fields and a pairwise field space is generated. Finally, we construct a classifier to predict site-ligand interactions in the pairwise field space.

\section{Materials and methods}

\subsection{Data set and data representations}

In this work, we use the data set constructed in our preliminary work [21]. There are totally 836 targets and 2710 corresponding ligands. Among 
those proteins, there are 782 targets with one binding site, which interact with 1988 ligands and form 2561 interaction pairs. The others possess multisites, which interact with 722 ligands and form 854 interaction pairs. The negative site-ligand pairs are generate as our preliminary work, totally 6830 target-ligand pairs include in our data set $[22,23]$.

Just as in our preliminary work [21], we represent each binding site as a 199-dimensional vector, each element in which denotes the occurring frequency of a set of trimers in the binding site (a trimer is a three-residues fragment of the binding site, and all possible trimers are clustered into 199 types according to their physical-chemical properties by Nagamine and Sakakibara[24, 25]); we represent each ligand as a 413-dimensional binary vector, each element corresponds to the presence/absence of one chemical substructure (fragment) in the ligand dictionary (413 substructures in all).

\subsection{Multi-fields interaction model}

Suppose there are $p$ binding sites, and $\boldsymbol{s}_{\boldsymbol{i}}$, an $m$-dimensional vector $(m=$ 199), denotes the $\boldsymbol{i}$-th binding site. Suppose there are $q$ ligands, and $\boldsymbol{l}_{\boldsymbol{j}}$, a $n$-dimensional vector $(n=413)$, denotes the $\boldsymbol{j}$-th ligand. We use $\boldsymbol{D}=$ $\left(\left(i_{1}, j_{1}\right),\left(i_{2}, j_{2}\right), \cdots,\left(i_{N}, j_{N}\right)\right)$ to denote all the site-ligand pairs in the data set, where $N$ is the total number of site-ligand pairs $(N=6830), i_{k} \in$ $\{1,2,3, \cdots, p\}, j_{k} \in\{1,2,3, \cdots, q\}$ and $\left(i_{k}, j_{k}\right)$ represents the $\boldsymbol{k}$-th siteligand pair. The binding states of $\boldsymbol{D}$ are labeled as $\boldsymbol{y}=\left(y_{1}, y_{2}, \cdots, y_{N}\right)$, where $y_{k} \in\{0,1\}$ represents binding label of the $\boldsymbol{k}$-th site-ligand pair.

Based on the multi-fields interaction hypothesis, there are multi-fields around a binding site and ligand, and the interaction is mediated by the fields. Since both the sites and ligands are broken into fragments, each field of sites/ligands can be considered as the combination of the fragment's corresponding field. We first deduce a single field interaction and other fields could be obtained accordingly.

\subsubsection{Single field calculation}

We regard each single-field interaction as the result of attraction/repulsion of a homogeneous field between the site and ligand fragments. For a given field, suppose $\boldsymbol{u}=\left(u_{1}, u_{2}, u_{3}, \ldots, u_{m}\right)^{T}$ be the field map of binding site fragments $\left(u_{k}\right.$ represents a fictitious field intensity generated by the $k$-th trimer cluster), and $\boldsymbol{v}=\left(v_{1}, v_{2}, v_{3}, \ldots, v_{n}\right)^{T}$ be the field map of ligand fragments $\left(v_{k}\right.$ represents a fictitious field intensity generated by the $k$-th chemical substructure). Then the interaction intensity of all interaction pairs in this field can 
be expressed as Equation (1).

$$
\sum_{(i, j) \in \boldsymbol{D}} d(i, j)\left(\boldsymbol{s}_{\boldsymbol{i}}^{T} \boldsymbol{u}\right)\left(\boldsymbol{l}_{\boldsymbol{j}}^{T} \boldsymbol{v}\right)=\boldsymbol{u}^{\boldsymbol{T}}\left(\sum_{(i, j) \in \boldsymbol{D}} d(i, j) \boldsymbol{s}_{\boldsymbol{i}} \boldsymbol{l}_{\boldsymbol{j}}^{T}\right) \boldsymbol{v}
$$

where $d(i, j)$ represents the binding confidence. If site $i$ can interact with ligand $j$ (observed in the data, i.e., the positive pair), we set $d(i, j)=1$. If we do not know whether site $i$ can interact with ligand $j$ (negative pair), we set $d(i, j)=-\lambda(0<\lambda<1)$. Because there might be binding events in the negative pairs, we have lower confidence in the negative bindings. We denote $\boldsymbol{A}=\sum_{(i, j) \in \boldsymbol{D}} d(i, j) \boldsymbol{s}_{\boldsymbol{i}} \boldsymbol{l}_{\boldsymbol{j}}^{T}$. Obviously, A is a constant matrix, and the total interaction intensity of the given field is $\boldsymbol{u}^{\boldsymbol{T}} \boldsymbol{A} \boldsymbol{v}$. Similar to the maximum likelihood estimation, we could find the field map vectors $\boldsymbol{u}$ and $\boldsymbol{v}$ by maximizing $\boldsymbol{u}^{\boldsymbol{T}} \boldsymbol{A} \boldsymbol{v}$, i.e.,

$$
\begin{aligned}
\max _{u, v}: & \boldsymbol{u}^{\boldsymbol{T}} \boldsymbol{A} \boldsymbol{v} \\
\text { s.t. } & \|\boldsymbol{u}\|_{2}^{2}=1, \quad\|\boldsymbol{u}\|_{1} \leq c_{1} \\
& \|\boldsymbol{v}\|_{2}^{2}=1, \quad\|\boldsymbol{v}\|_{1} \leq c_{2}
\end{aligned}
$$

For feasibility, we constrain the L2-norm of $\boldsymbol{u}$ and $\boldsymbol{v}$ to be one. Now that a fragment in the sites/ligands usually has a certain kind of propensity, for example, the electrostatic/polar field around a hydrophobic fragment would be weak, $\boldsymbol{u}$ and $\boldsymbol{v}$ are expected to be sparse. To achieve this goal, we introduce the L1-norm to control sparseness. Thus the L1-norm of $\boldsymbol{u}$ and $\boldsymbol{v}$ are set smaller than $c_{1}$ and $c_{2}$ respectively, where $c_{1}$ and $c_{2}$ are two parameters controlling sparse levels of $\boldsymbol{u}$ and $\boldsymbol{v}$ respectively. Equation (2) can be solved by using Algorithm 1. In every iteration of Algorithm 1, we use Algorithm 2, which is based on Karush-Kuhn-Tucker method, to compute the correspondent $\boldsymbol{u}$ (step 6) and $\boldsymbol{v}$ (step 8) respectively.

\subsubsection{Multiple fields calculation}

Using Algorithm 1 and 2, we can obtain one homogeneous field map pair $\boldsymbol{u}, \boldsymbol{v}$ and the corresponding $\rho\left(\boldsymbol{u}, \boldsymbol{v}\right.$ and $\rho$ also know as $\boldsymbol{u}_{\mathbf{1}}, \boldsymbol{v}_{\mathbf{1}}$ and $\rho_{1}$ respectively). Nevertheless, the interaction between a target and a ligand is the comprehensive result of multiple fields. Suppose there are $\Gamma$ fields involved in the site-ligand interaction, and $\boldsymbol{u}_{\gamma}$ represents the $\boldsymbol{\gamma}$-th target field map vector $(\boldsymbol{\gamma} \leq \Gamma)$; $\boldsymbol{v}_{\boldsymbol{\gamma}}$ represents the $\boldsymbol{\gamma}$-th ligand field map vector; the map vector pair $\left(\boldsymbol{u}_{\gamma}, \boldsymbol{v}_{\gamma}\right)$ derives the $\boldsymbol{\gamma}$-th homogeneous field. We assume that different types of fields are heterogeneous and heterogeneous fields can 


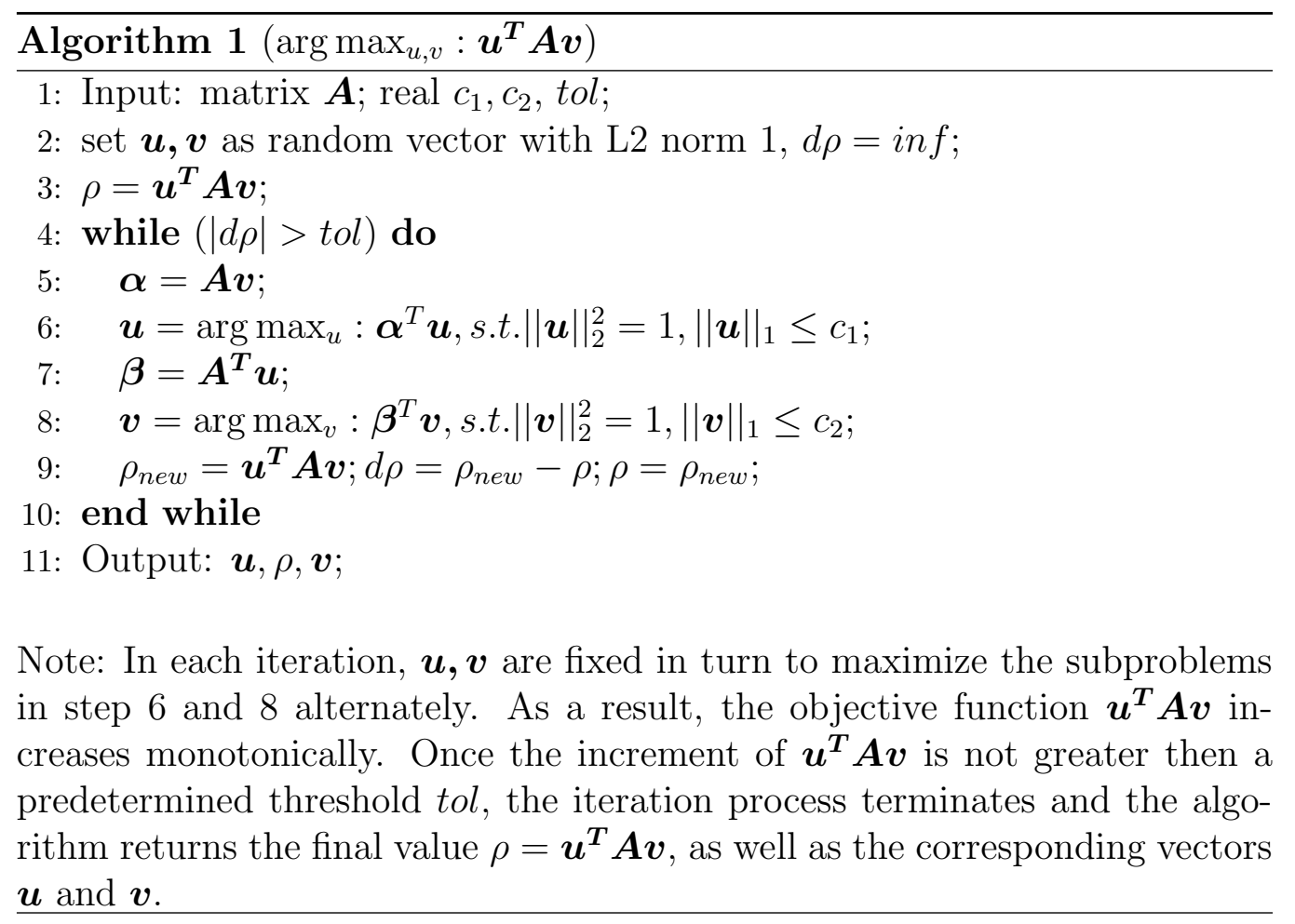

not interact with each other, for instance, the electrostatic field does not interact with hydrophobic field. Therefore, we can set the heterogeneous fields to be orthogonal. That means $\boldsymbol{u}_{\boldsymbol{i}}^{T} \boldsymbol{u}_{\boldsymbol{j}}=0$ and $\boldsymbol{v}_{\boldsymbol{i}}^{T} \boldsymbol{v}_{\boldsymbol{j}}=0$ if $i \neq j$, $(i, j \in\{1,2, \cdots, \Gamma\})$. As a result, any pair of $\left(\boldsymbol{u}_{\gamma}, \boldsymbol{v}_{\gamma}\right)$ could be solved according to Equation (3).

$$
\begin{aligned}
\max _{u_{\gamma}, v_{\gamma}}: & \boldsymbol{u}_{\gamma}^{\boldsymbol{T}} \boldsymbol{A} \boldsymbol{v}_{\boldsymbol{\gamma}} \\
\text { s.t. } & \left\|\boldsymbol{u}_{\boldsymbol{\gamma}}\right\|_{2}^{2}=1, \quad\left\|\boldsymbol{u}_{\boldsymbol{\gamma}}\right\|_{1} \leq c_{1} \\
& \left\|\boldsymbol{v}_{\boldsymbol{\gamma}}\right\|_{2}^{2}=1, \quad\left\|\boldsymbol{v}_{\gamma}\right\|_{1} \leq c_{2} \\
& \boldsymbol{u}_{\boldsymbol{i}}^{\boldsymbol{T}} \boldsymbol{u}_{\boldsymbol{\gamma}}=0, \quad i=1,2, \cdots, \gamma-1 \\
& \boldsymbol{v}_{\boldsymbol{j}}^{\boldsymbol{T}} \boldsymbol{v}_{\boldsymbol{\gamma}}=0, \quad j=1,2, \cdots, \gamma-1
\end{aligned}
$$

Directly solving Equation (3) is difficult. Let $\boldsymbol{A}_{\boldsymbol{\gamma}}=\boldsymbol{A}-\sum_{i=1}^{\gamma-1} \rho_{i} \boldsymbol{u}_{\boldsymbol{i}} \boldsymbol{v}_{\boldsymbol{i}}^{T}$. Because of the orthogonality, we have $\boldsymbol{u}_{\gamma}^{\boldsymbol{T}} \boldsymbol{A} \boldsymbol{v}_{\gamma}=\boldsymbol{u}_{\gamma}^{\boldsymbol{T}} \boldsymbol{A}_{\gamma} \boldsymbol{v}_{\gamma}+\boldsymbol{u}_{\gamma}^{\boldsymbol{T}}\left(\sum_{i=1}^{\gamma-1} \rho_{i} \boldsymbol{u}_{\boldsymbol{i}} \boldsymbol{v}_{\boldsymbol{i}}^{T}\right) \boldsymbol{v}_{\gamma}=$ $\boldsymbol{u}_{\gamma}^{\boldsymbol{T}} \boldsymbol{A}_{\gamma} \boldsymbol{v}_{\gamma}$, then Equation (3) can be rewritten as following,

$$
\begin{aligned}
\max _{u_{\gamma}, v_{\gamma}}: & \boldsymbol{u}_{\gamma}^{\boldsymbol{T}} \boldsymbol{A}_{\boldsymbol{\gamma}} \boldsymbol{v}_{\boldsymbol{\gamma}} \\
\text { s.t. } & \left\|\boldsymbol{u}_{\boldsymbol{\gamma}}\right\|_{2}^{2}=1, \quad\left\|\boldsymbol{u}_{\boldsymbol{\gamma}}\right\|_{1} \leq c_{1} \\
& \left\|\boldsymbol{v}_{\boldsymbol{\gamma}}\right\|_{2}^{2}=1, \quad\left\|\boldsymbol{v}_{\boldsymbol{\gamma}}\right\|_{1} \leq c_{2}
\end{aligned}
$$




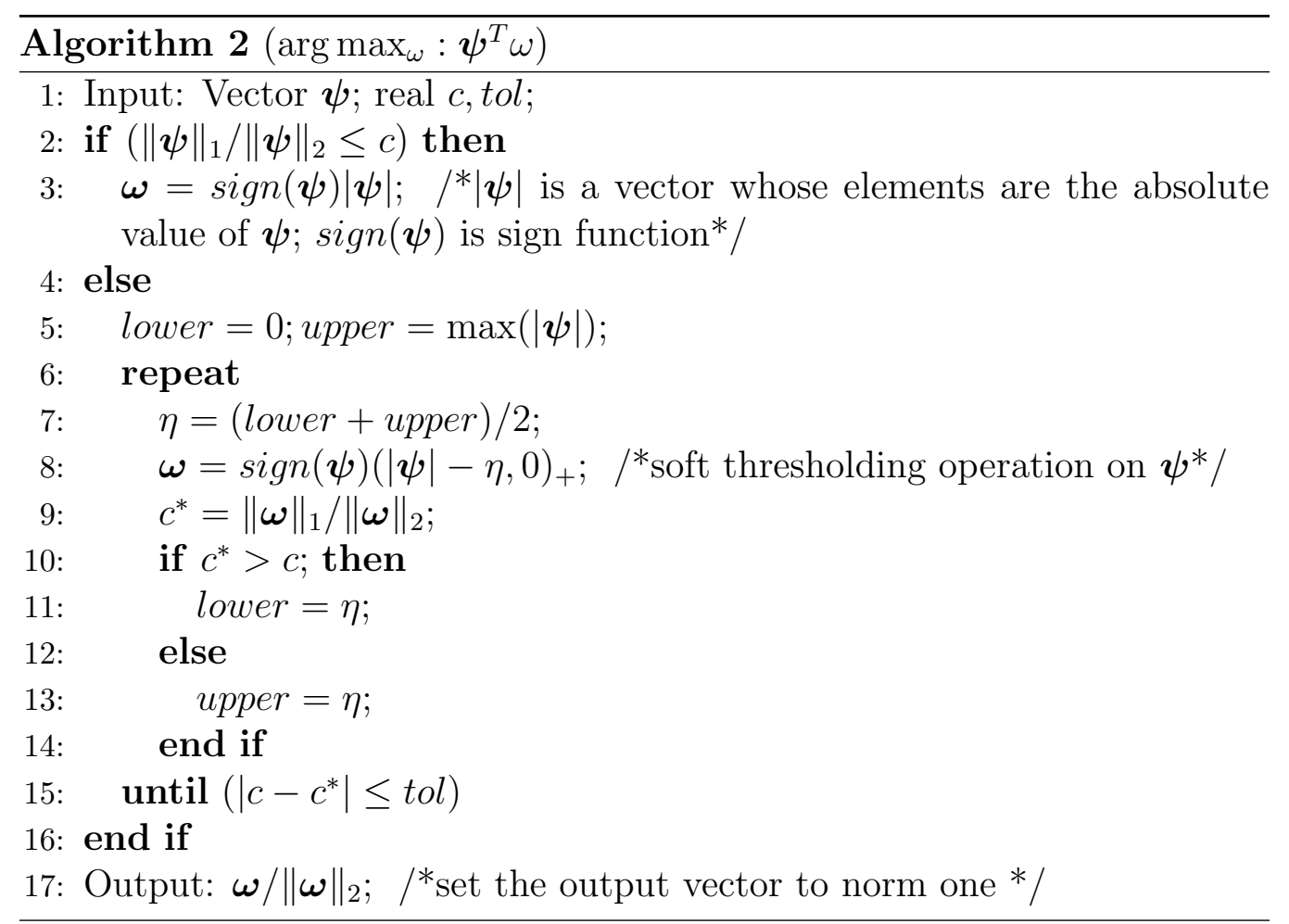

Equation (4) is similar with Equation (2), which means that we can solve out every $\left(\boldsymbol{u}_{\boldsymbol{\gamma}}, \boldsymbol{v}_{\boldsymbol{\gamma}}\right)$ and the corresponding $\rho_{\gamma}$ with Algorithm 1 and 2. Although Equation (4) is not completely equal with Equation (3), it generates similar results. All the site and ligand map vectors constitute the target and ligand map matrices $\boldsymbol{U}$ and $\boldsymbol{V}$ respectively (Figure 1).

\subsubsection{Prediction of target-ligand interaction}

Just described as Section 2.2.2, we can get multiple field maps $\left(\boldsymbol{u}_{\boldsymbol{\gamma}}, \boldsymbol{v}_{\boldsymbol{\gamma}}\right)$ and the corresponding $\rho_{\gamma}$. Therefore, for the pair of target $i$ and ligand $j$, the interaction intensity raised by $\left(\boldsymbol{u}_{\gamma}, \boldsymbol{v}_{\gamma}\right)$ can then be calculated by Equation (5).

$$
f_{\gamma}(i, j)=\boldsymbol{\rho}_{\gamma}\left(\boldsymbol{s}_{\boldsymbol{i}}^{\boldsymbol{T}} \boldsymbol{u}_{\boldsymbol{\gamma}}\right)\left(\boldsymbol{l}_{\boldsymbol{j}}^{\boldsymbol{T}} \boldsymbol{v}_{\gamma}\right)
$$

By this way, a pair $(i, j)$ in $\boldsymbol{D}$ can be transformed into a uniform field vector $\left(f_{1}(i, j), \ldots, f_{\gamma}(i, j), \ldots f_{\Gamma}(i, j)\right)$. Based on the vector and its corresponding label in $\boldsymbol{y}$, we can use logistic regression (LR) to construct the classification model, MFIM, to predict the interaction between target $i$ and ligand $j$. 
According to logistics regression, we can get

$$
L R(i, j)=\frac{1}{1+e^{-\sum_{\gamma=1}^{\Gamma} \alpha_{\gamma} f_{\gamma}(i, j)}}
$$

where $\alpha_{\gamma}$ are the parameters of logistics regression and $\sum_{\gamma=1}^{\Gamma} \alpha_{\gamma} f_{\gamma}(i, j)=$ $\boldsymbol{s}_{\boldsymbol{i}}^{\boldsymbol{T}}\left(\sum_{\gamma=1}^{\Gamma} \alpha_{\gamma} \rho_{\gamma} \boldsymbol{u}_{\boldsymbol{\gamma}} \boldsymbol{v}_{\gamma}^{\boldsymbol{T}}\right) \boldsymbol{l}_{\boldsymbol{j}}$. Let $\boldsymbol{N}=\sum_{\gamma=1}^{\Gamma} \alpha_{\gamma} \rho_{\gamma} \boldsymbol{u}_{\boldsymbol{\gamma}} \boldsymbol{v}_{\gamma}^{\boldsymbol{T}}$. For binding site $i$ and ligand $j$, we can infer whether they interact with each other according to

$$
\operatorname{Pred}_{L R}(i, j)= \begin{cases}1 & \boldsymbol{s}_{\boldsymbol{i}}^{\boldsymbol{T}} \boldsymbol{N} \boldsymbol{l}_{j} \geq 0 \\ 0 & \boldsymbol{s}_{\boldsymbol{i}}^{\boldsymbol{T}} \boldsymbol{N} \boldsymbol{l}_{\boldsymbol{j}}<0\end{cases}
$$

From Equation (7), we can see that the matrix $\boldsymbol{N}$ actually stands for a weighted network, where fragments of binding sites (rows) and ligands (columns) are two kinds of nodes, and the interactions between two kinds of nodes are the edges. Every edge is weighted by the fragment interaction intensities. Once $\boldsymbol{N}$ has been extracted from the training data, it can be used to predict the interaction between any pair of target and ligand available.

All the experiments were performed on a desktop (Lenovo, i3 CPU, 4G RAM, Windows 7), and the algorithmic procedures, learning the field maps and the logistic regression, were finished within one minute.

\section{Results}

Before demonstrating the concrete results, we want to show super parameters selection and optimization. $c_{1}$ and $c_{2}$ are two super parameters controlling sparse level of the field map vectors. Smaller $c_{1}$ and $c_{2}$ are harmful to the orthogonality of $\boldsymbol{U}$ and $\boldsymbol{V}$ as well as the AUC score. To guarantee the orthogonality of the field map vectors as well as the high AUC score, we set $c_{1}=0.8$ and $c_{2}=0.7$ in this work. The super parameter $\Gamma$ indicates the number of field. We could choose $\Gamma$ by imitating QSAR, and five or six named fields are selected, such as electrostatic field, hydrophobic field and hydrogen bond field. However the fields in MFIM are very different from those in QSAR, and we choose to figure out the number of fields by experiment. With the increasing number of fields, the AUC score first increases quickly and then reaches a platform. With high AUC score, we choose fewer fields. Thus we select 50 fields in this work. (The figures and tables supporting the super parameters optimization are shown in the Supplementary Material.) 


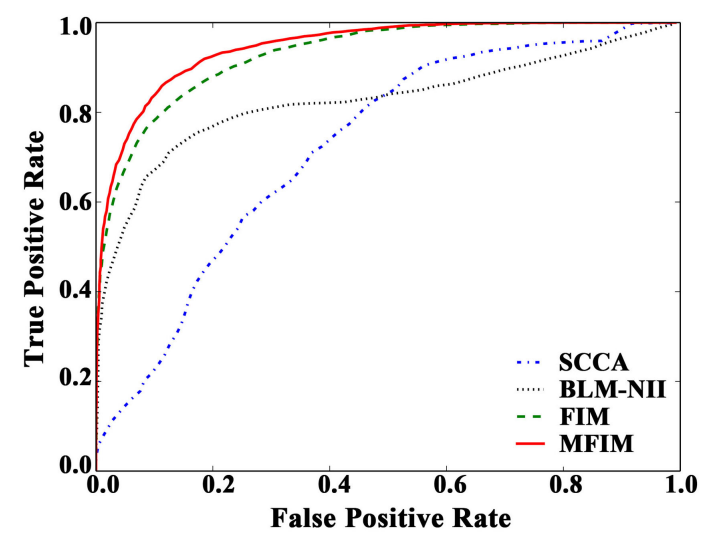

Figure 2: ROC curves for MFIM, MIF, SCCA and BLM-NII

In the following part of this section, we first illustrate prediction performance of MFIM, and then analyze the fragment interaction network in detail to reveal its properties and the underlying chemical insights. Moreover, we provide a list of the top 1000 predicted target-ligand interactions in the Supplementary Material.

\subsection{Performance evaluation of MFIM}

In order to the evaluation, we compare the performance of MFIM with our preliminary work [21], as well as other two approaches: BLM-NII [6] and SCCA [26]. BLM-NII is chosen for the reason that it displays the state-ofthe-art performance on Yamanishi's data set, which is viewed as the "Gold Standard". While SCCA is chosen due to the fact that it also has the ability to find the potential inherent factors governing the target-ligand interactions, just as our models. BLM-NII and SCCA were constructed based on the same data sets, but different with our data set; Moreover, the data sets used for BLM-NII and SCCA were smaller than ours. To be fair, we implement all models based on our data set. The performances are evaluated by multiple criteria, such as accuracy (ACC, the percentage of correct predictions), precision (the percentage of true positive instances in all predicted positive predictions), recall (the percentage of predicted true positive predictions in all true positive instances) and area under receiver operating characteristic curve (AUC, comprehensive evaluation of classifier performance, between 0.5 to 1, the larger the better). Using five-folds CV test, the performances of all methods are shown in Table 1. 
Table 1: Statistics of the prediction performance

\begin{tabular}{lcccc}
\hline & ACC & Precision & Recall & AUC \\
\hline SCCA & 0.565 & 0.552 & 0.621 & 0.799 \\
BLM-NII & 0.727 & 0.712 & 0.812 & 0.858 \\
FIM & 0.835 & 0.848 & 0.821 & 0.916 \\
MFIM & 0.873 & 0.870 & 0.876 & 0.953 \\
\hline
\end{tabular}

Table 1 shows that the ACC and AUC scores of SCCA are $56.5 \%$ and $79.9 \%$ respectively, which means the correct prediction rate is only slightly higher than random guess (the expected correct rate of random guess is $50 \%$ ) and the comprehensive performance is not good. We guess that the poor performance of SCCA is due to lacking of powerful classifier and it only serves as a feature extraction approach. The BLM-NII performs well on our data set. The AUC score of BLM-NII is $85.8 \%$. The BLM-NII is sensitive to the topology structure of the target-ligand network. Since it builds classifiers for each target and each ligand, the nodes with larger degree are in favour of prediction. We have performed the target-ligand network topology structure analysis in our preliminary work, the target-ligand network based on our data set is more spare than that in Yamanishi's "Gold Standard" data set, which could give us some insight of lower AUC score of BLM-NII in our data set and the proposed method could resist to data sparse. The AUC score of FIM is $91.6 \%$, which is consistent with our previous work. The logistic regression (LR) based on MFIM performs excellently in our data set. The AUC score of LR is $95.3 \%$, which is much higher than that of SCCA and BLM-NII. Figure 2 demonstrates detailed ROC curves for MFIM, FIM, SCCA and BLM-NII. It is obvious that MFIM outperforms the SCCA and BLM-NII.

\subsection{Fragment interaction network}

By convention, it is the time to analyze the relationship between the field map vectors and the chemical properties of the site and ligand fragments. However, it is difficult to quantify the chemical properties of a fragment. We analyze the fragment interaction network and the chemical mechanisms underlying instead.

\subsubsection{Network stability analysis}

Since the edge weights in the network $\boldsymbol{N}$ represent the fragment interaction intensities, they should be objective and robust to the parameter fluctuation. The super parameter $\lambda$ represents the confidence of the negative 

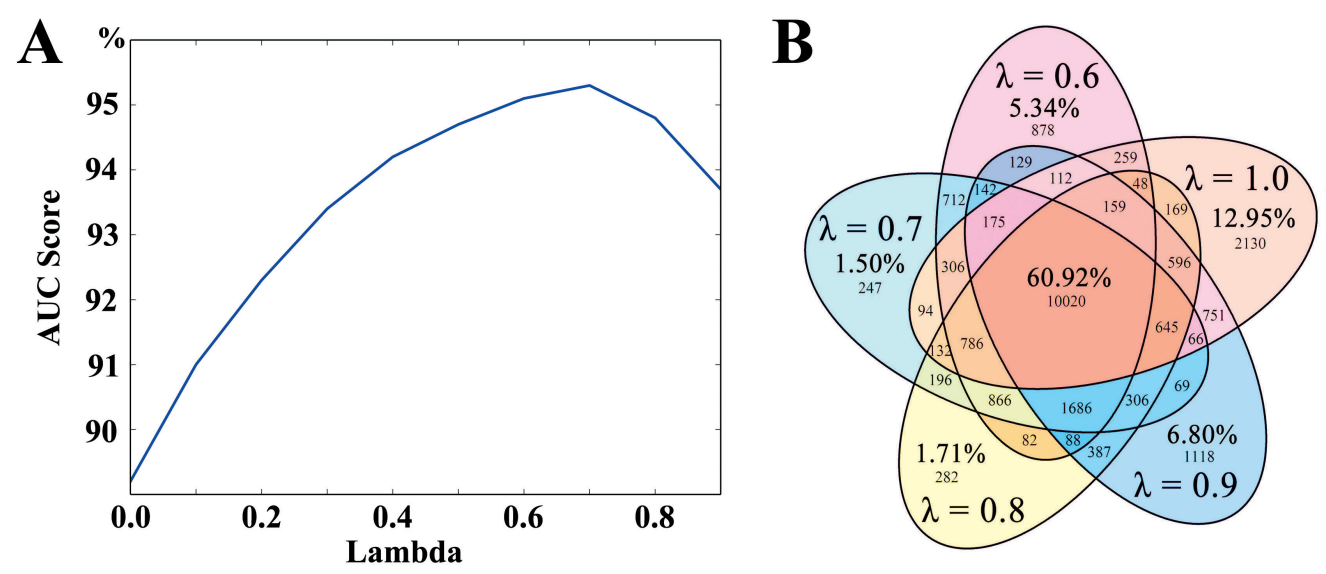

Figure 3: The analysis of $\lambda$. A) The chosen of $\lambda$. B) The overlap of the top $20 \%$ interactions with different $\lambda$.

interaction pairs. Different $\lambda$ may generate different fragment-fragment interaction matrices, $\boldsymbol{N}$, and lead to different prediction performances. Before analyzing the network stability, a proper $\lambda$ should be chosen. And then, around the chosen $\lambda$, we attempt to explore the network stability. The curve of five-fold cross validation AUC score with respect to $\lambda$ shown in Figure 3/A. With increasing $\lambda$, the AUC score first increases and then decreases. The best performance achieves at $\lambda=0.7$, therefore we choose $\lambda=0.7$ in this work. Analyzing the network stability is difficult for the real network is unavailable to us. Nevertheless, if the interactions in networks obtained with different $\lambda$ values are significantly overlapped, it is expected that the fragment interaction network should be stable and insensitive to the parameter value. Therefore, we investigate the overlap between the top 20\% (16448) strong interactions of the networks obtained with $\lambda$ varying from 0.6 to 1.0, and the result shows in Figure 3/B. From which we can see that more than $60 \%$ of the 16448 interactions are overlapped, implying the stability of the fragment interaction network. The pairwise Wilcoxon test of the distribution of the overlapped 10020 interaction intensities with $\lambda=0.6, \cdots, 1.0$ (totally $C_{5}^{2}=10$ pairwise Wilcoxon tests) also shows that the difference is insignificant (with a significance level of 0.01), which suggests the stability of the fragment interaction network once again. 

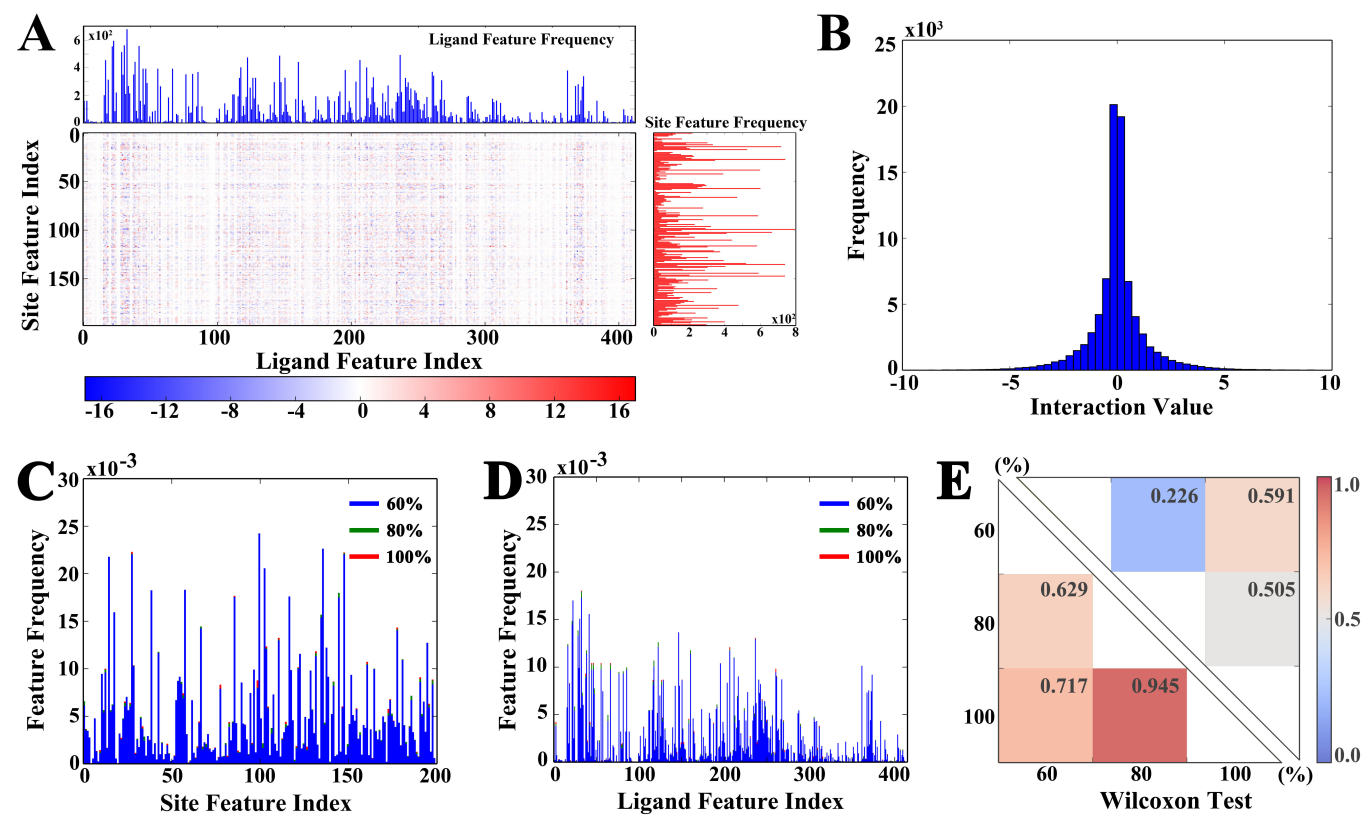

Figure 4: The overview of fragment interaction network. A) The center box is the fragment interaction network $(\lambda=0.7)$, and the top (blue) and right (red) side are the ligand and site fragment frequency distribution respectively. B) The histogram of fragment interaction values. Most of the values are around zero. C) The site fragment frequency distribution of $60 \%, 80 \%$ and all sites. D) The ligand fragment frequency distribution of $60 \%, 80 \%$ and all ligands. E) The $\mathrm{p}$ values of Wilcoxon test between the site/ligand fragment frequency distribution of $60 \%, 80 \%$ and all samples. The upper and lower parts are p values of site and ligand fragment frequency distribution respectively.

\subsubsection{Network overview}

An obvious feature of the fragment interaction network (Figure 4/A) is that the weight of the connections is either positive or negative. The positive values may suggest that the fragment pairs prefer to interact with each other, while the negative values may suggest that the fragment pairs prefer not. This characteristic is very useful for drug design such as the lead compound optimization, where one can only consider the high (positive) affinity chemical substructures binding with the target fragments.

Another obvious feature of the network is that the connections are sparse and most of the interaction values are around zero (Figure 4/B), which mean that one target fragment could only recognize a small number of ligand fragments, and vice versa The sparse connections could reflect the specificity 
Table 2: The selected target and ligand features and their potential interaction mechanism

\begin{tabular}{|c|c|c|c|}
\hline Target Features & Ligand Features & $\begin{array}{c}\text { Interaction } \\
\text { Values } \\
\end{array}$ & Potential Mechanism \\
\hline TF131:L(I, F* V , ... ) & LF30:C $[\mathrm{NH}] \mathrm{c}$ & 11.75 & stacked $\pi-\pi$ conjugate \\
\hline TF151:D*(I, L, V, ... ) & LF372:c1cnnc1 & 9.92 & $\begin{array}{l}\text { polar interaction } \\
\text { hydrogen bond }\end{array}$ \\
\hline TF57:I* $(Y, F, V, \ldots)$ & LF30:C $[\mathrm{NH}] \mathrm{c}$ & -8.30 & polar-nonpolar repulsion \\
\hline TF116:A* $(\mathrm{E}, \mathrm{H}, \mathrm{M}, \ldots)$ & LF29:c[NH2] & -8.34 & polar-nonpolar repulsion \\
\hline
\end{tabular}

Note: The first letter of target features is the center amino acid and the letters in the parenthesis represent the high frequency subordinate amino acids. The ligand feature is represented by smarts (SMiles ARbitrary Target Specification). The last column shows the potential mechanism of the target and ligand fragments. The amino acids with star are chosen to show in the sub-figures in the last column.

during the target-ligand binding process. We are surprised with the sparse connections, because we do not impose any sparsity on the connections. One possible explanation is that the sparsity of the network originates in the sparsity of the original interaction data, the fragment frequency of sites and ligands. To check this possibility, we calculate the frequency distribution of ligand and site fragments and demonstrate the results on the top and right side of the network respectively. It is obvious that the site fragment of low frequency corresponds the whiter row (smaller interaction values) of the feature interaction matrix, while the site fragment of high frequency corresponds the colorful row (significant interaction values). Similar results are observed with the ligand fragments, but the impact is on the corresponding columns. In short, either the site or ligand fragment with low frequency leads to smaller interaction values (sparse connections) and the sparsity of the network mainly originates in the sparsity of the original interaction data. The sparsity of the original interaction data usually indicates the lacking of samples. However, the evolution might select the fragment distribution, and increasing samples would not change the fragment distribution much. We calculate the site fragment frequency distribution of $60 \%, 80 \%$ and all sites, and the results imply that with the increasing samples the site fragment fre- 
quency distributions almost remain stable (Figure 4/C). Similar results from the ligand fragment frequency distributions are shown in Figure 4/D. The p values of pairwise Wilcoxon test between the site fragment frequency distribution of $60 \%, 80 \%$ and all sites are shown in the upper part of Figure 4/E, while those of the ligand fragment frequency distribution are shown in the lower part. All the $\mathrm{p}$ values are larger than 0.01 , which indicate the differences between those distributions are insignificant and increasing samples would not change the fragment distribution much. In a word, the evolution might select the special fragment distribution, which could lead to the sparsity of the fragment interaction network.

\subsubsection{Chemical mechanism analysis}

The induced network $\boldsymbol{N}$ can not only be used to predict the targetligand interactions via Equation (7), but also reflect the chemical interaction mechanisms between fragments. In other words, the positive values may suggest that the fragment pairs prefer to attracting each other, while the negative values may imply that the fragment pairs prefer to repulsing. To analyze the interaction mechanism, we first select the top 50 positive and top 50 negative interactions from the overlapped 10020 significant ones. The selected interactions form a small subnetwork, form which we choose the maximal connected subgraph to analyze the mechanism. Finally, we list a part of the detailed potential interaction mechanisms in Table 2 (The full table shown in the Supplementary Material). From this table, for instance, we can see that ligand fragment 30 (LF30) trends to bind to target fragment 131 (TF131); and the potential chemical mechanism could be that the phenyl in phenylalanine (F, the high frequency amino acid in TF131) forms stacked $\pi-\pi$ conjugate interaction with the phenyl in LF30. While ligand fragment 30 (LF30) is not in favor of binding to target fragment 57 (TF57); and the potential reason could be that the polar nitrogen in LF30 repulses the hydrophobic residue isoleucine (I, the center main amino acid ) in TF57. Other interactions in Table 2 could be analyzed accordingly.

\subsubsection{Case study}

We can derive the mechanism of a target-ligand interaction via the fragment interaction network. Taking the p38 mitogen-activated protein kinases (MAPK, also named cytokinin specific binding protein, CSBP) as an example here. p38 MAP Kinases (MAPK) are a class of mitogen-activated protein kinases which participate in a signaling cascade controlling cellular responses 

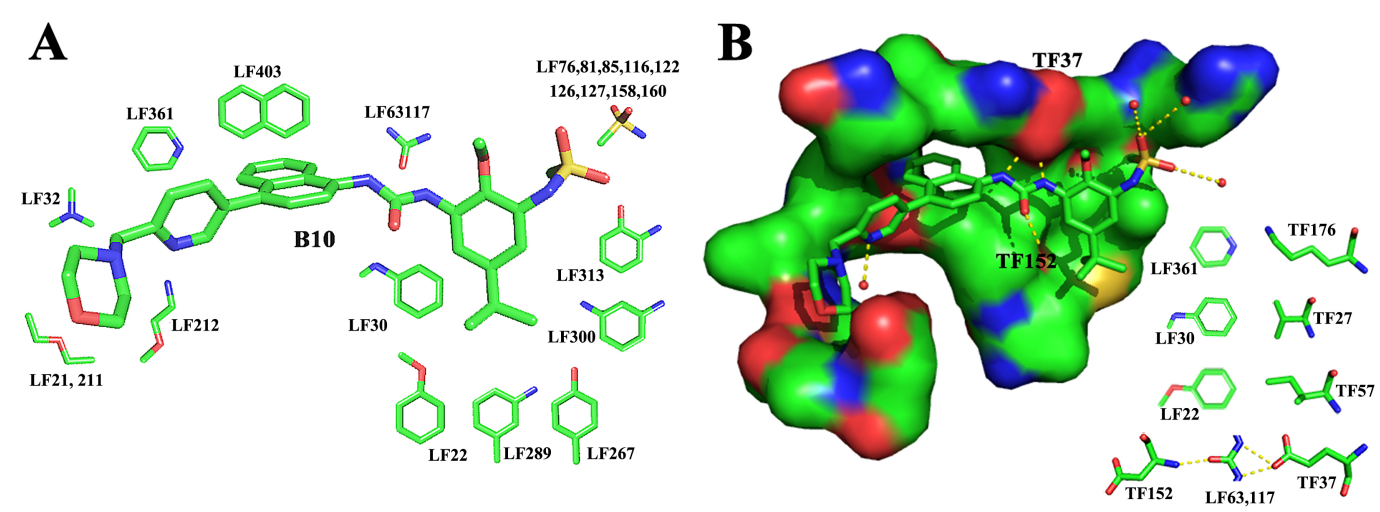

Figure 5: A) B10 (a N-Phenyl-N'-Naphthylurea analogue) and its substructures. B) The complex of B10 and MAPK. The green, red, yellow and blue represent carbon, oxygen and sulfur and oxide respectively. The yellow dash lines represent hydrogen bonds. The red balls represent water.

to cytokines and stress [27]. B10 (a N-Phenyl-N'-Naphthylurea analogue) is a ligand of p38 MAP Kinases. p38 MAP Kinases possess a hydrophobic binding pocket (the green in Figure 5/B) with a few polar amino acids, such as glutamate (71E, TF37) and aspartate (168D, TF152). B10 bind p38 MAP Kinases main through hydrophobic interaction and hydrogen bonds with $71 \mathrm{E}$ and 168D (B10 also can from hydrogen bonds with water[28]). To check whether the fragment interactions in 3GI3 (PDB ID) reflect the reported interactions, we break B10 and binding site into fragments. Figure 5/A shows that B10 is well represented, and totally get 22 ligand fragments are obtained. Ligand feature 63/117 (the two features are overlapped) can interaction with target feature TF37 and TF152, the interaction values are 1.42 and 1.15 respectively. The positive interaction values indicate that the interaction among LF117, TF37 and TF152 are in favor of binding, which is in line with the Cirillo's report. Moreover, three high intensity hydrophobic interactions are fund, LF361-TF176, LF30-TF27, LF22-TF57 (Figure 5).

\section{Discussion and conclusions}

In this work, a new model called multi-fields interaction model (MFIM) is proposed. MFIM emphasizes the basic chemical interactions between amine acids and ligand fragments. Concretely, we break the sites and ligands into fragments based on the predefined dictionaries, and then extract fields from the fragments. A field could interact with its homogeneous field, and a 
pairwise field space is generated. The comparison result shows that MFIM outperforms other two representative approaches. More importantly, our method can provide a fragment interaction network. Although the prediction of target-ligand interactions involves in filling a never-ending targetligand table [3], our induced (limited) network can infer the never-ending target-ligand via Equation (7). Furthermore, the network could help us understand the interaction mechanisms between fragments, thus can help to find potential ligand fragments for a given target site.

The fragment interaction network may be useful in drug design. In that setting, if we know the ligand-binding site, we could break it into fragments based on the target dictionary. Then, the network could help us to find potential ligand fragments with high binding affinities, which could improve the drug design efficiency and save a lot of time and resources. Moreover, we no longer take the target as a whole, but extract and describe the ligandbinding sites, by which we could predict site-ligand interactions. The high accuracy allows it to serve as an optional step previous to molecular docking.

However, there are a few drawbacks. First, MFIM requires 3D structure of protein which may limit it application in practice. Second, we break the binding site and ligand into fragments without including the spatial arrangement information.

In conclusion, MFIM is a chemically interpretable model and outperforms other two prevalent approaches on our data set. The fragment interaction network could make us understand how the fragments interact with others and help us find potentially high affinity ligand fragments for a given site.

\section{Acknowledgement}

This work was supported by the National Science Foundation of China [61272274, 60970063, 31270101, 61402340]; the program for New Century Excellent Talents in Universities [NCET-10-0644]; Natural Science Foundation of Hubei Province of China [2014CFB194]; the Ministry of Science and Technology of China (973 and 863 Programs) and the National Mega Project on Major Drug Development.

\section{References}

[1] M. Kanehisa, From genomics to chemical genomics: new developments in kegg, Nucleic Acids Res. 34(Database issue) (2006) D354-D357. 
[2] B. Stockwell, Chemical genetics: ligand-based discovery of gene function, Nat. Rev. Genet. 1 (2000) 116-125.

[3] D. Rognan, Chemogenomic approaches to rational drug design, Br. J. Pharmacol. 152 (2007) 38-52.

[4] Y. Yamanishi, Prediction of drug-target interaction networks from the integration of chemical and genomic spaces, Bioinformatics 24 (2008) i232-i240.

[5] D. Kitchen, Docking and scoring in virtual screening for drug discovery: methods and applications, Nat. Rev. Drug Discov. 3 (2004) 935-949.

[6] K. Bleakley, Y. Yamanishi, Supervised prediction of drug-target interactions using bipartite local models, Bioinformatics 25 (2009) 2397-2403.

[7] S. N. T. van Laarhoven, E. Marchiori, Gaussian interaction profile kernels for predicting drug-target interaction, Bioinformatics 27 (2011) 3036-3043.

[8] Z. Xia, Semi-supervised drug-protein interaction prediction from heterogeneous biological spaces, BMC Syst. Biol. 4(Suppl 2) (2010) S6.

[9] J. Mei, Drug-target interaction prediction by learning from local information and neighbors, Bioinformatics 29 (2013) 238-245.

[10] C. Leslie, Mismatch string kernels for discriminative protein classification, Bioinformatics 20 (2004) 467-476.

[11] C. Yanover, T. Hertz, Predicting protein-peptide binding affinity by learning peptidepeptide distance functions, RECOMB - (2005) 456-471.

[12] E. H. B. Chen, R. Guha, Pubchem as a source of polypharmacology, J. Chem. Inf. Model. 49 (2009) 2044-2055.

[13] J.-P. Vert, B. Scholkopf, Local alignment kernels for biological sequences., kernel methods in computational biology, MIT Press - (2004) $131-154$.

[14] L. Jacob, J. Vert, Protein-ligand interaction prediction: an improved chemogenomics approach, Bioinformatics 24 (2008) 2149-2156. 
[15] T. Smith, S. Waterman, Identification of common molecular subsequences, J. Mol. Biol 147 (1981) 195-197.

[16] Z. Y. Yongcui Wang, N. Deng, Computationally probing drugprotein interactions via support vector machine, Letters in Drug Design and Discovery 7 (2010) 370-378.

[17] N. D. Yongcui Wang, Chunhua Zhang, Y. Wang, Kernel based data fusion improves the drug-protein interaction prediction, Computational Biology and Chemistry 35 (2011) 353-362.

[18] S. C. Yongcui Wang, Naiyang Deng, Y. Wang, Computational study of drugs by integrating omics data with kernel methods, Molecular Informatics 32 (2013) 930-941.

[19] S. Zhao, S. Li, Network-based relating pharmacological and genomic spaces for drug target identification, PLoS ONE 5 (2010) e11764.

[20] C. Nantasenamat, A practical overview of quantitative structure-activity relationship, Excli. J. 8 (2009) 74-88.

[21] C. Wang, J. Liu, Predicting target-ligand interactions using protein ligand-binding site and ligand substructures, BMC Syst. Biol. 9(Suppl 1) (2015) $\mathrm{S} 2$.

[22] D. Wheeler, Database resources of the national center for biotechnology information, Nucleic Acids Res. 35 (2007) D5-D12.

[23] D. R. J. Meslamani, E. Kellenberger, sc-pdb: a database for identifying variations and multiplicity of 'druggable' binding sites in proteins, Bioinformatics 27 (2011) 1324-1326.

[24] N. Nagamine, Y. Sakakibara, Statistical prediction of protein chemical interactions based on chemical structure and mass spectrometry data, Bioinformatics 23 (2007) 2004-2012.

[25] M. Venkatarajan, W. Braun, New quantitative descriptors of amino acids based on multidimensional scaling of a large number of physicalchemical properties, J. Mol. Model 7 (2001) 445-453. 
[26] Y. Yamanishi, Extracting sets of chemical substructures and protein domains governing drug-target interactions, J. Chem. Inf. Model. 51 (2011) 1183-1194.

[27] J. Han, R. Ulevitch, A map kinase targeted by endotoxin and hyperosmolarity in mammalian cells, Science 265 (1994) 808-811.

[28] P. Cirillo, E. Hickey, Discovery and characterization of the n-phenyln'-naphthylurea class of p38 kinase inhibitors, Bioorganic \& Medicinal Chemistry Letters 19 (2009) 2386-2391. 